\title{
Cordycepin inhibits lipopolysaccharide-induced cell migration and invasion in human colorectal carcinoma HCT-116 cells through down-regulation of prostaglandin $\mathrm{E}_{2}$ receptor EP4
}

\author{
Jin-Woo Jeong ${ }^{1}$, Cheol Park ${ }^{2}$, Hee-Jae Cha ${ }^{3}$, Su Hyun Hong ${ }^{4,5}$, Shin-Hyung Park ${ }^{6}$, Gi-Young Kim ${ }^{7}$, Woo Jean Kim ${ }^{8}$, \\ Cheol Hong Kim ${ }^{2}$ Kyoung Seob Song ${ }^{10, *}$ E Yung Hyun Choi ${ }^{4,5, *}$ \\ ${ }^{1}$ Freshwater Bioresources Utilization Bureau, Nakdonggang National Institute of Biological Resources, Sangju $17104,{ }^{2}$ Department of \\ Molecular Biology, College of Natural Sciences, Dongeui University, Busan 47340, ${ }^{3}$ Department of Parasitology and Genetics, Kosin \\ University College of Medicine, Busan 49267, ${ }^{4}$ Department of Biochemistry, Dong-Eui University College of Korean Medicine, Busan \\ 47227, ${ }^{5}$ Anti-Aging Research Center, Dong-Eui University, Busan 47340, ${ }^{6}$ Department of Pathology, Dong-Eui University College of \\ Korean Medicine, Busan 47227, ${ }^{7}$ Department of Marine Life Sciences, Jeju National University, Jeju 63243, ${ }^{8}$ Department of Anatomy, \\ Kosin University College of Medicine, Busan 49267, ${ }^{9}$ Department of Pediatrics, Sungkyunkwan University Samsung Changwon Hospital, \\ Changwon 51353, ${ }^{10}$ Department of Physiology, Kosin University College of Medicine, Busan 49267, Korea
}

Prostaglandin $\mathrm{E}_{2}\left(\mathrm{PGE}_{2}\right)$, a major product of cyclooxygenase-2 (COX-2), plays an important role in the carcinogenesis of many solid tumors, including colorectal cancer. Because $\mathrm{PGE}_{2}$ functions by signaling through $\mathrm{PGE}_{2}$ receptors (EPs), which regulate tumor cell growth, invasion, and migration, there has been a growing amount of interest in the therapeutic potential of targeting EPs. In the present study, we investigated the role of EP4 on the effectiveness of cordycepin in inhibiting the migration and invasion of HCT116 human colorectal carcinoma cells. Our data indicate that cordycepin suppressed lipopolysaccharide (LPS)-enhanced cell migration and invasion through the inactivation of matrix metalloproteinase (MMP)-9 as well as the down-regulation of COX-2 expression and $\mathrm{PGE}_{2}$ production. These events were shown to be associated with the inactivation of EP4 and activation of AMP-activated protein kinase (AMPK). Moreover, the EP4 antagonist AH23848 prevented LPS-induced MMP-9 expression and cell invasion in HCT116 cells. However, the AMPK inhibitor, compound C, as well as AMPK knockdown via siRNA, attenuated the cordycepin-induced inhibition of EP4 expression. Cordycepin treatment also reduced the activation of CREB. These findings indicate that cordycepin suppresses the migration and invasion of HCT116 cells through modulating EP4 expression and the AMPK-CREB signaling pathway. Therefore, cordycepin has the

*Corresponding authors. Kyoung Seob Song, Tel: +82-51-990-6236; Fax: +82-51-990-3081; E-mail: kssong@kosin.ac.kr; Yung Hyun Choi, Tel: +82-51-850-7413; Fax: +82-51-853-4036; E-mail: choiyh@ deu.ac.kr

https://doi.org/10.5483/BMBRep.2018.51.10.120

Received 28 May 2018, Revised 29 June 2018, Accepted 3 September 2018

Keywords: AMPK, Cell migration/invasion, Cordycepin, CREB, EP4 potential to serve as a potent anti-cancer agent in therapeutic strategies against colorectal cancer metastasis. [BMB Reports 2018; 51(10): 532-537]

\section{INTRODUCTION}

Cordyceps species are rare herbs that are occasionally used in traditional medicine $(1,2)$. They have been used to treat various diseases, such as cancer, and are also known to have anti-tumor properties, the ability to scavenge free radicals, and immune-stimulating abilities (3-5). Cordycepin is the main bioactive component in Cordyceps species such as Cordyceps militaris and C. sinensis $(2,4)$. Cordycepin has many biological properties, including inflammation inhibition (6), platelet aggregation inhibition (7), anti-tumor effects (8), and chondrocyte hypertrophy inhibition (9). However, the molecular mechanism through which cordycepin inhibits cancer cell migration and invasion remains unclear.

The physiological functions and signaling of prostaglandin $E_{2}\left(P G E_{2}\right)$ are related to the activation of EP receptors (EP1-4), which are G-protein-coupled receptors (GPCRs) (10). PGE $_{2 \text {, }}$ which is regulated by cyclooxygenase- 2 (COX-2), promotes cell proliferation and the invasion of colorectal tumors (11). Signaling through the EP2 receptor activates the protein kinase A (PKA) pathway, which induces the phosphorylation of cyclic adenosine monophosphate (CAMP) response element binding protein (CREB) in the gastrointestinal tract (12). However, the molecular mechanism through which this intracellular mediator is related to cell invasion and migration in colorectal cancer remains unclear, along with the anti-inflammatory effects of $\mathrm{PGE}_{2}$ in colorectal cancer. Identifying the intracellular signaling mechanism that mediates cell invasion and movement, which in turn mediate the effects of $\mathrm{PGE}_{2}$, is

ISSN: 1976-670X (electronic edition)

Copyright (c) 2018 by the The Korean Society for Biochemistry and Molecular Biology

(c) This is an open-access article distributed under the terms of the Creative Commons Attribution Non-Commercial License (http://creativecommons.org/licenses/by-nc/4.0) which permits unrestricted non-commercial use, distribution, and reproduction in any medium, provided the original work is properly cited. 
critical to understanding the main properties of colorectal cancer and developing effective therapies.

AMP-activated protein kinase (AMPK) is a well-conserved serine/threonine protein kinase containing a catalytic subunit $(\alpha)$ and two regulatory subunits ( $\beta$ and $\gamma$ ) that is expressed in many tissues (13). Some studies have suggested that AMPK can function as a tumor suppressor by altering inflammation and causing cell-cycle arrest during tumorigenesis $(14,15)$. In addition, when activated by 5 -aminoimidazole-4-carboxamideribonucleoside (AICAR) or phenformin, AMPK induces cell death through the mitogen-activated protein kinases pathway $(16,17)$. In addition, AMPK is mediated to Kahweol-induced glucose uptake in mouse embryo fibroblast cells (18). Taken together, these findings suggest that AMPK activation may be useful in controlling cell death in colorectal cancer cells.
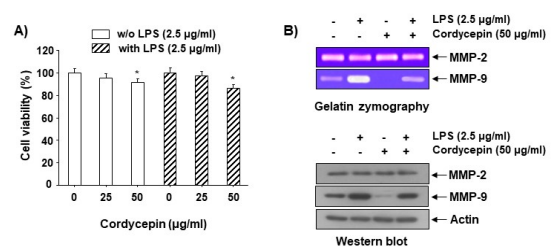

c)
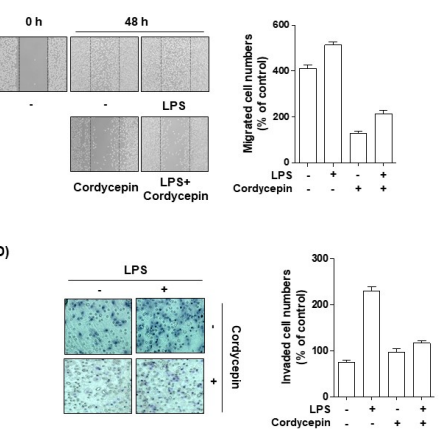

Fig. 1. Inhibitory effects of cordycepin on the migration and invasion of human colorectal carcinoma HCT116 cells. (A) Cells were incubated with varying concentrations of cordycepin in the absence or presence of LPS for $48 \mathrm{~h}$ in serum-free medium, and proliferation was determined using an MTT assay. The data are expressed as the mean $\pm \mathrm{SD}$ of triplicate experiments. $* \mathrm{P}<$ 0.05 compared to control. (B) Cells at $80 \%$ confluence were treated with various concentrations of cordycepin in serum-free medium. The conditioned medium was collected after $48 \mathrm{~h}$, and gelatin zymography was performed in triplicate. Representative blots are shown. Equal amounts of cellular proteins were separated on SDS-polyacrylamide gels and transferred to PVDF membranes. The membranes were probed with specific antibodies and visualized using an ECL. Actin was used as an internal control. (C) Cells were scratched with a pipette tip and treated with cordycepin or LPS for $48 \mathrm{~h}$. Migrated cells were imaged using phase-contrast microscopy. (D) The cells were plated onto the apical sides of Matrigel-coated filters in serum-free medium. Medium containing $20 \%$ FBS was placed in the basolateral chamber to act as a chemoattractant. After $48 \mathrm{~h}$, cells on the apical side were removed using a Q-tip. The cells on the bottom of the filter were then stained using $\mathrm{H} \& \mathrm{E}$ and photographed.
Because cordycepin can greatly affect the pathogenesis of colorectal disease, we hypothesized that cordycepin downregulates EP4 expression and its downstream signaling functions in human colorectal cancer cells. In order to examine the signaling pathway involved, we performed in vitro human cell-based assays. Our results suggest that cordycepin inhibits cell invasion and migration in lipopolysaccharide (LPS)-treated HCT-116 cells via the EP4-AMPKCREB axis. These pathways provide new insights into the molecular mechanism of cell invasion and may reveal novel targets for therapeutic medications.

\section{RESULTS}

\section{Cordycepin inhibits LPS-induced cell migration and invasion in HCT116 human colorectal carcinoma cells}

In order to investigate the pharmacological potential of cordycepin on LPS-induced cell migration and invasion, we first determined the dose dependence of the cytotoxic effects of cordycepin in the absence or presence of LPS for $48 \mathrm{~h}$ in HCT116 cells using an MTT assay. Cordycepin at 25-50 $\mu \mathrm{g} / \mathrm{ml}$ did not show any cytotoxic effect on HCT-116 cells with or without $2.5 \mu \mathrm{g} / \mathrm{ml}$ LPS (Fig. 1A). Therefore, a concentration of cordycepin within this range was employed in the remaining experiments. We next used gelatin zymography and Western blot analyses to investigate the inhibitory effects of cordycepin on the activation and expression of matrix metalloproteinase (MMP) proteins. Interestingly, compared to LPS alone,
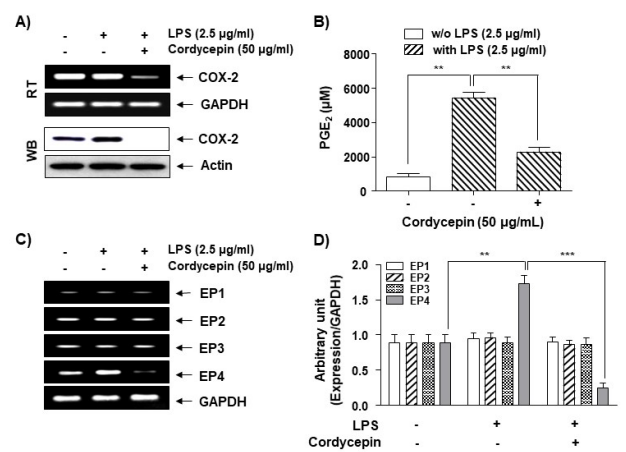

Fig. 2. Inhibitory effects of cordycepin on $\mathrm{PGE}_{2}$ production and expression of COX-2 and EPs in LPS-stimulated HCT-116 cells. Cells were pre-treated with $50 \mu \mathrm{g} / \mathrm{ml}$ of cordycepin for $1 \mathrm{~h}$ prior to incubation with LPS for $48 \mathrm{~h}$. Total RNA (A; upper panel) and proteins (A; lower panel) were prepared and used for the RT-PCR analysis of COX-2 gene expression with GAPDH as an internal control. Actin was used as an internal control. (B) The culture supernatants were isolated, and the amount of $\mathrm{PGE}_{2}$ production was determined. The data are expressed as the mean $\pm \mathrm{SD}$ of three independent experiments. ${ }^{* * P}<0.01$ compared to LPS alone. (C) Total RNA was prepared and used for RT-PCR analysis of EP1-4 gene expression. (D) The data represent the EP1-4 mRNA levels in the LPS-treated cultures compared with control cultures from three independent experiments $(* * P<0.01$, $* * * P<0.001)$. 
cotreatment with both cordycepin and LPS inhibited the activation and expression of MMP-9, but had no effect on MMP-2 activity or expression (Fig. 1B). In vitro invasion and migration assays were used to investigate the inhibitory effects of cordycepin on the invasive potency of LPS-treated HCT116 cells. As shown in Fig. 1C and D, LPS-stimulated cell migration and cell invasion were significantly inhibited by cordycepin. These results suggest that nontoxic concentrations of cordycepin have an inhibitory effect on the invasiveness of LPS-treated HCT-116 cells.

\section{Cordycepin inhibits the expression of COX-2 and EP4 and the production of $\mathrm{PGE}_{2}$ in LPS-treated HCT116 cells}

Previous reports have shown that cell migration and invasion increase the activation of the EPs as well as the expression of the COX-2 product $\mathrm{PGE}_{2}$ in several cancer cell lines (17-20). Thus, we investigated whether the EP4-COX-2-PGE 2 cascade affects LPS-induced cell migration and invasion in HCT116 cells. While LPS did not alter the COX-2 mRNA level, it slightly increased the COX2 protein level (Fig. 2A). However, cordycepin dramatically inhibited LPS-induced COX-2 mRNA and protein expression. Cordycepin also showed an inhibitory effect on LPS-induced $\mathrm{PGE}_{2}$ production at a concentration of $50 \mu \mathrm{g} / \mathrm{ml}$, as demonstrated by enzyme immunoassays (Fig. 2B), suggesting that the inhibitory effects of cordycepin on LPS-induced $\mathrm{PGE}_{2}$ production are controlled by COX-2 gene expression. In addition, we attempted to identify which of the $\mathrm{PGE}_{2}$ receptor subtypes is responsible for the LPS-mediated production of $\mathrm{PGE}_{2}$ in HCT-116 cells. Interestingly, only EP4 mRNA expression was affected by cordycepin, while EP2, EP3, and EP4 were not affected (Fig. 2C, D; Supplement Table 1). These results suggest that cordycepin inhibits EP4 and COX-2 expression to regulate $\mathrm{PGE}_{2}$ production in $\mathrm{HCT} 116$ cells.

\section{Cordycepin inhibits MMP9-mediated cell migration and invasion}

In order to determine whether cordycepin could inhibit LPS-mediated MMP9 expression via EP4, we used AH23848, an EP4 antagonist. It dramatically inhibited LPS-induced MMP-9 expression, but not MMP-2 expression (Fig. 3A, B). In addition, cell migration and invasion assays were performed in order to examine whether cordycepin could inhibit cell invasion and migration in LPS-treated cells through inhibiting the EP4 receptor. While AH23848 inhibited LPS-mediated cell migration and invasion (Fig. 3C, D), overexpressed EP4 partially increased both of these (Supplement Fig. 1). These results indicate that cordycepin inhibited LPS-mediated cell migration and invasion via MMP and EP4.

Cordycepin-activated AMPK inhibits EP4 gene expression in LPS-treated cells and Cordycepin decreases CREB activation by inhibiting EP4 gene expression

AMPK is related to many physiological phenomena, such as cell death $(21,22)$, autophagy (23), and metabolic control (24).
In particular, AMPK can control cell polarity, migration, and cytoskeletal dynamics (25). As shown in Fig. 4A, the phosphorylation levels of AMPK and acetyl-CoA carboxylase (ACC), a downstream target kinase of AMPK, were increased by cordycepin treatment in a time-dependent manner, suggesting that AMPK signaling was activated. In order to determine whether cordycepin-activated AMPK can affect EP4 expression, we used compound C, an AMPK inhibitor. Cordycepin strongly inhibited EP4 gene expression (Fig. 4C), and compound $\mathrm{C}$ restored EP4 gene expression in LPS-treated cells. We employed COX-2 activity assay and $\mathrm{PGE}_{2}$ ELISA assay in order to determine whether compound $C$ could partially restore COX-2 activity and $\mathrm{PGE}_{2}$ production. While cordycepin decreased LPS-mediated COX-2 activity and $\mathrm{PGE}_{2}$ production, compound $\mathrm{C}$ slightly increased both of these in LPS-treated cells (Supplement Fig. 2). The results from a genetic study using AMPK-specific siRNA (Fig. 4D) were the same as those from the pharmaceutical study (Fig. 4C), suggesting that cordycepin inhibits EP4 gene expression via the AMPK pathway in LPS-treated cells. In addition, many studies have reported that CREB may be a down-stream protein of the AMPK pathway $(26,27)$. Thus, we identified CREB activation in LPS-treated cells. Cordycepin dramatically inhibited CREB activation in LPS-treated cells in a time-dependent manner (Fig. 4E). H89, an inhibitor of PKA and MSK, was used to inhibit CREB activation (28). As expected, H89 strongly inhibited EP4 gene expression by inhibiting CREB activation in LPS-treated cells (Fig. 4F), indicating that CREB may be critical for EP4 gene expression. In order to determine whether cordycepin could inhibit
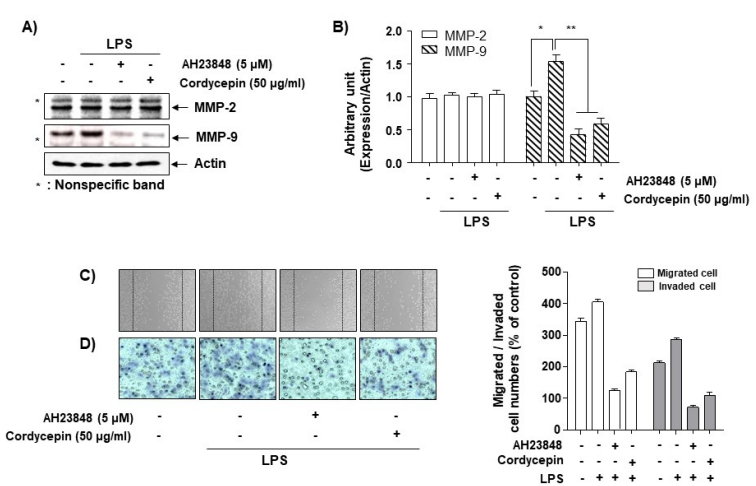

Fig. 3. Increase in cordycepin-induced anti-invasiveness by the inhibition of EP4 signaling in HCT-116 cells. (A, B) Cells were pretreated with LPS $(2.5 \mu \mathrm{g} / \mathrm{ml})$ for $1 \mathrm{~h}$ prior to treatment with AH23848 or cordycepin $(50 \mu \mathrm{g} / \mathrm{ml})$ for $48 \mathrm{~h}$. The cells were lysed, and equal amounts of protein were subjected to Western blotting. (C, D) Cells were pretreated with LPS $(2.5 \mu \mathrm{g} / \mathrm{ml})$ for 1 $h$ before being challenged with $\mathrm{AH} 23848$ or cordycepin (50 $\mu \mathrm{g} / \mathrm{ml}$ ) for $6 \mathrm{~h}$. The cell migration (C) and Matrigel invasion (D) assays were performed. The experiment was repeated three times, and similar results were obtained. 

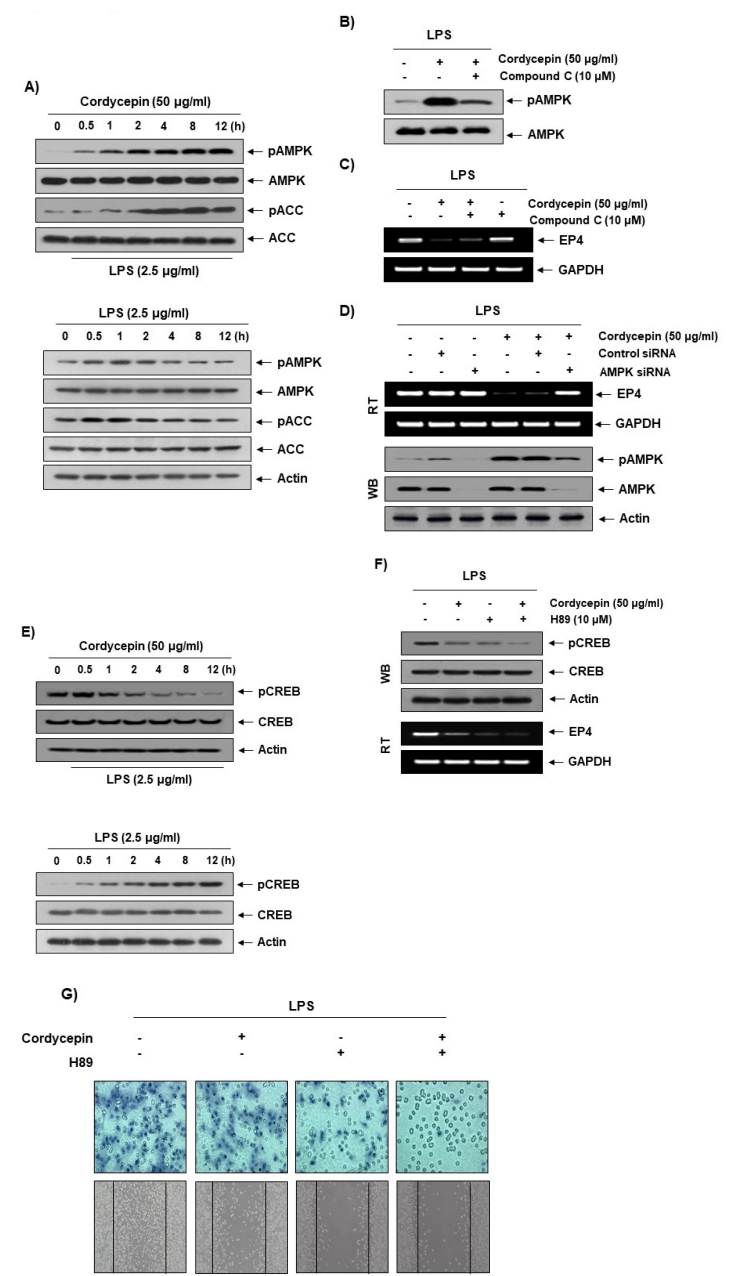

Fig. 4. Involvement of the AMPK pathway in the reduction of EP4 expression by cordycepin. (A; upper panel) Cells were pretreated with LPS $(2.5 \mu \mathrm{g} / \mathrm{ml})$ for $1 \mathrm{~h}$ prior to treatment with cordycepin (50 $\mu \mathrm{g} / \mathrm{ml}$ ) for the indicated times. (A; lower panel) Cells were treated with LPS $(2.5 \mu \mathrm{g} / \mathrm{ml})$ for the indicated times. Immunoblotting analyses were performed with anti-pAMPK, anti-AMPK, anti-pACC, and anti-ACC antibodies. (B) The effects of cordycepin and compound $\mathrm{C}$ on AMPK activation in LPS-stimulated HCT-116 cells. Cells were pretreated with LPS $(2.5 \mu \mathrm{g} / \mathrm{ml})$ for $1 \mathrm{~h}$ prior to treatment with either cordycepin $(50 \mu \mathrm{g} / \mathrm{ml})$ only or both cordycepin and compound $\mathrm{C}(10 \mu \mathrm{M})$ for $12 \mathrm{~h}$. (C) The effects of cordycepin and compound C on EP4 expression in LPS-stimulated cells. Cells were pretreated with LPS $(2.5 \mu \mathrm{g} / \mathrm{ml})$ for $1 \mathrm{~h}$ prior to treatment with either cordycepin $(50 \mu \mathrm{g} / \mathrm{ml})$ only or both cordycepin and compound $\mathrm{C}(10 \mu \mathrm{M})$ for $12 \mathrm{~h}$. (D) The cells were transfected with AMPK small interfering (siRNA) or control siRNA for $24 \mathrm{~h}$. The transfected cells were treated with $50 \mu \mathrm{g} / \mathrm{ml}$ cordycepin for $48 \mathrm{~h}$. (E; upper panel) Cells were pretreated with LPS $(2.5 \mu \mathrm{g} / \mathrm{ml})$ for $1 \mathrm{~h}$ prior to treatment with cordycepin (50 $\mu \mathrm{g} / \mathrm{ml}$ ) for the indicated times. ( $\mathrm{E}$; lower panel) Cells were treated with LPS $(2.5 \mu \mathrm{g} / \mathrm{ml})$ for the indicated times. Cells were pretreated with LPS $(2.5 \mu \mathrm{g} / \mathrm{ml})$ for $1 \mathrm{~h}$ prior to treatment with either cordycepin $(50 \mu \mathrm{g} / \mathrm{ml})$ only or both cordycepin and H89 $(10 \mu \mathrm{M})$ for $12 \mathrm{~h}$. Then, we performed Western blot and RT-PCR (F) as well as cell migration and invasion assay $(\mathrm{G})$.
LPS-mediated cell migration and invasion via CREB activation, we performed these assays. Cotreatment with cordycepin and H89 was shown to dramatically inhibit cell migration and invasion compared to cordycepin alone in LPS-treated cells (Fig. 4G). These results suggest that cordycepin strongly decreases CREB-mediated cell migration and invasion through the alteration of EP4 gene expression in LPS-treated cells.

\section{DISCUSSION}

In this study, we examined the anti-cell migration and invasion effects of cordycepin in LPS-treated human colorectal carcinoma HCT-116 cells. Cordycepin had no cytotoxic effects on HCT-116 cells at the concentrations tested (Fig. 1), but it did show anti-inflammatory activity. Previous studies have shown that cordycepin has anti-cancer effects in human breast cancer cells (29), human lung cancer cells (30), oral squamous cell carcinoma (31), and human bladder cancer cells (32). Cordycepin has also been shown to have anti-cancer effects in human colorectal cancer cells, but there have been few reports on cordycepin's role in altering early events (i.e., migration and invasion) to prevent the progression into cell transformation. Because most of the literature on cordycepin's anti-tumor effects has focused thus far on cell apoptosis, the effects of cordycepin on early events, like cell migration and invasion during tumorigenesis, remain a matter of speculation. In particular, there have been no reports until now that cordycepin inhibits LPS-induced cell migration and invasion in human colorectal cancer cells, although cordycepin has been found to decrease tumor necrosis factor- $\alpha$-induced cell migration and invasion in human bladder cancer cells (33). The major difference in these pathways is the function of the EP4 receptor versus the mediator of intracellular signaling nuclear factor- $\mathrm{KB}$. This is a critical finding, because it is the first stage of signal transduction at the receptor as an early event.

Understanding the physiological characteristics of the EP4 receptor could provide further insight into the intracellular signaling mechanism that leads to protein complex formation of G-protein coupled receptors (34). Cordycepin decreased gene expression of the EP4 receptor, but not that of EP1, -2, or -3 , and also reduced $\mathrm{PGE}_{2}$ production (Fig. 2C, D). This is unsurprising, because EP4 is preferentially expressed in the heart and intestines and expressed to a lesser degree in the lungs, kidneys, and brain (35). EP4 is a Gos protein that stimulates CAMP production, and cordycepin dramatically increased AMPK activation (Fig. 4B) and significantly inhibited EP4 gene expression, suggesting that AMPK can specifically inhibit EP4 gene expression. Funahashi et al. (36) reported that EP-induced $\mathrm{PGE}_{2}$ expression abolished AMPK activity through protein kinase A signaling transmission. Reciprocally, a cordycepin-induced increase in AMPK activity could inhibit EP4 activity, thus preventing its physiological signaling. Taken together, these data suggest that LPS increases EP4 activation 
to produce $\mathrm{PGE}_{2}$, which induces cell migration and invasion by inhibiting AMPK, and that cordycepin inhibits this signaling mechanism, giving it anti-cancer properties that affect the early tumorigenesis stages.

Cordycepin inhibits CREB signaling in order to regulate its physiological functions. CREB-related gene expression is important for microenvironmental homeostasis under pathological conditions, and CREB has been considered as a major target of novel drugs. As seen in Fig. $4 \mathrm{E}$, cordycepin inhibited LPS-induced CREB activation, and both H89 and forskolin induced CRE activity, suggesting that cordycepin decreased CREB activation via the EP4-AMPK pathway in order to prevent cell migration and invasion in HCT-116 cells.

In conclusion, our results demonstrate that the levels of CREB activation were dramatically decreased by the negative regulation of AMPK expression through the deactivation of EP4 in cordycepin-treated HCT116 cells. This study provides novel insights into the molecular mechanisms of cell migration in HCT-116 cells. Controlling the expression of the EP4 receptor may be a new strategy for preventing cell migration and invasion of HCT-116 cells. This study also highlights the potential for the therapeutic use of cordycepin in the treatment of colorectal cancer.

\section{MATERIALS AND METHODS}

\section{Reagents}

Cordycepin (MW, 251.2; Product No. C3394) from C. militaris, LPS (Escherichia coli 026:B6), Griess reagent, Tween-20, bovine serum albumin (BSA), H89, forskolin, compound C, and 3-(4,5-dimethylthiazol-2-yl)-2, 5-diphenyltetrazolium bromide (MTT) were purchased from SigmaAldrich Chemical Co. (St. Louis, MO, USA). Antibodies against COX-2, MMP-2, MMP-9, AMPK, pAMPK, ACC, pACC, CREB, pCREB, and actin were purchased from Santa Cruz Biotechnology (Santa Cruz, CA, USA). The peroxidase-labeled donkey anti-rabbit immunoglobulin, peroxidase-labeled sheep anti-mouse immunoglobulin, and enhanced chemiluminescence (ECL) detection kit were purchased from Amersham Corp. (Arlington Heights, IL, USA). The enzyme-linked immunosorbent assay (ELISA) kit for $\mathrm{PGE}_{2}$ was obtained from R\&D Systems (Minneapolis, MN, USA). COX-2, EP1-4, and glyceraldehyde-3-phosphate dehydrogenase (GAPDH) oligonucleotide primers were purchased from Bioneer (Seoul, Korea). All other chemicals were purchased from Sigma-Aldrich Chemical Co.

\section{Cell culture and MTT assay}

HCT-116 human colon cancer cells were obtained from the American Type Culture Collection (Manassas, VA, USA). These cells were cultured in RPMI-1640 medium supplemented with $10 \%$ fetal bovine serum (Gibco BRL, Gaithersburg, MD, USA) and $1 \%$ penicillin-streptomycin at $37^{\circ} \mathrm{C}$ in a humid environment containing $5 \% \mathrm{CO}_{2}$. For the cell viability assay,
HCT-116 cells were seeded in 6-well plates at a density of $2 \times$ $10^{5}$ cells per well. After $24 \mathrm{~h}$ of stabilization, the cells were treated with various concentrations of cordycepin for $1 \mathrm{~h}$, then stimulated with LPS $(2.5 \mu \mathrm{g} / \mathrm{ml})$ for an additional $48 \mathrm{~h}$. Cell viability was determined using the MTT assay, which is based on the conversion of MTT to MTT-formazan by mitochondrial enzymes.

\section{In vitro wound-healing assay}

HCT-116 cells were seeded in 6-well plates and grown overnight to confluence. The monolayers were then scratched with a 200- $\mu$ l pipette tip in order to create a wound and washed twice with serum-free RPMI-1640 so as to remove floating cells; the medium was then replaced with serum-free medium supplemented with cordycepin baicalein in the presence or absence of LPS. The rate of wound closure was assessed and imaged $48 \mathrm{~h}$ later. Each value is derived from three randomly selected fields.

\section{Matrigel invasion assay}

HCT-116 cells were incubated in RPMI-1640 with $10 \%$ FBS and collected via trypsinization. Cells $\left(2 \times 10^{5}\right.$ cells/well $)$ in serum-free medium were added to the inner cup of a 24-well transwell chamber (Corning Life Sciences, Oneonta, NY, USA) that had been coated with $50 \mathrm{ml}$ of Matrigel (BD Biosciences, Franklin Lakes, NJ, USA; 1:10 dilution in serum-free medium). Medium supplemented with $10 \%$ serum or the indicated agent was then added to the outer cup. After $48 \mathrm{~h}$, cells that had migrated through the Matrigel and the 8-mm pore size membrane were fixed, stained with hematoxylin and eosin (H\&E, Sigma-Aldrich Chemical Co.), then photographed under an inverted microscope. Each experiment was performed in triplicate.

\section{ACKNOWLEDGEMENTS}

This study was supported by a grant from the National Research Foundation of Korea (NRF) funded by the Korea government (NRF-2016R1C1B1014724 for JWJ, 2016R1D1A 1B03932521 for KSS, and 2018R1A2B2005705 for YHC).

\section{CONFLICTS OF INTEREST}

The authors have no conflicting interests.

\section{REFERENCES}

1. Yue K, Ye M, Zhou Z et al (2013) The genus Cordyceps: a chemical and pharmacological review. J Pharm Pharmacol 65, 474-493

2. Das SK, Masuda M, Sakurai A et al (2010) Medicinal uses of the mushroom Cordyceps militaris: current state and prospects. Fitoterapia 81, 961-968

3. Paterson RR (2008) Cordyceps: a traditional Chinese medicine and another fungal therapeutic biofactory? 
Phytochemistry 69, 1469-1495

4. Buenz EJ, Bauer BA, Osmundson TW et al (2005) The traditional Chinese medicine Cordyceps sinensis and its effects on apoptotic homeostasis. J Ethnopharmacol 96, 19-29

5. Shrestha B, Tanaka E, Han JG et al (2014) A brief chronicle of the genus cordyceps fr., the oldest valid genus in cordycipitaceae (hypocreales, ascomycota). Mycobiology 42, 93-99

6. Choi YH, Kim GY and Lee HH (2014) Anti-inflammatory effects of cordycepin in lipopolysaccharide-stimulated RAW 264.7 macrophages through Toll-like receptor 4-mediated suppression of mitogen-activated protein kinases and NF-KB signaling pathways. Drug Des Devel Ther 8, 1941-1953

7. Cho HJ, Cho JY, Rhee MH et al (2007) Inhibitory effects of cordycepin (3'-deoxyadenosine), a component of Cordyceps militaris, on human platelet aggregation induced by thapsigargin. J Microbiol Biotechnol 17, 1134-1138

8. Nakamura K, Shinozuka K and Yoshikawa N (2015) Anticancer and antimetastatic effects of cordycepin, an active component of Cordyceps sinensis. J Pharmacol Sci $127,53-56$

9. Cao Z, Dou C, Li J et al (2016) Cordycepin inhibits chondrocyte hypertrophy of mesenchymal stem cells through PI3K/Bapx1 and Notch signaling pathway. BMB Rep 49, 548-553

10. Woodward DF, Jones RL, and Narumiya S (2011) International Union of Basic and Clinical Pharmacology. LXXXIII: classification of prostanoid receptors, updating 15 years of progress. Pharmacol Rev 63, 471-538

11. Greenhough A, Smartt HJ, and Moore AE (2009) The COX-2/PGE 2 pathway: key roles in the hallmarks of cancer and adaptation to the tumour microenvironment. Carcinogenesis 30, 377-386

12. Dey I, Lejeune $M$, and Chadee $K$ (2006) Prostaglandin $E_{2}$ receptor distribution and function in the gastrointestinal tract. Br J Pharmacol 149, 611-623

13. Jeon SM (2016) Regulation and function of AMPK in physiology and diseases. Exp Mol Med 48, e245

14. O'Neill LA and Hardie DG (2013) Metabolism of inflammation limited by AMPK and pseudo-starvation. Nature 493, 346-355

15. Li W, Saud SM, Young MR et al (2015) Targeting AMPK for cancer prevention and treatment. Oncotarget 6, 7365-7378

16. Petti C, Vegetti C, Molla A et al (2012) AMPK activators inhibit the proliferation of human melanomas bearing the activated MAPK pathway. Melanoma Res 22, 341-350

17. Kim JI, Lakshmikanthan V, Frilot N et al (2010) Prostaglandin $\mathrm{E}_{2}$ promotes lung cancer cell migration via EP4-betaArrestin1-c-Src signalsome. Mol Cancer Res 8, 569-577

18. Baek JH, Kim NJ, Song JK, Chun KH (2017) Kahweol inhibits lipid accumulation and induces Glucose-uptake through activation of AMP-activated protein kinase (AMPK). BMB Rep 50, 566-571

19. Kim MJ, Kim HS, Lee SH et al (2014) NDRG2 controls COX-2/PGE ${ }_{2}$-mediated breast cancer cell migration and invasion. Mol Cells 37, 759-765

20. Menter DG and Dubois RN (2012) Prostaglandins in cancer cell adhesion, migration, and invasion. Int J Cell Biol 2012, 723419

21. Gwinn DM, Shackelford DB, Egan DF et al (2008) AMPK phosphorylation of raptor mediates a metabolic checkpoint. Mol Cell 30, 214-226

22. Kalender A, Selvaraj A, Kim SY et al (2010) Metformin, independent of AMPK, inhibits mTORC1 in a rag GTPase-dependent manner. Cell Metab 11, 390-401

23. Egan DF, Shackelford DB, Mihaylova MM et al (2011) Phosphorylation of ULK1 (hATG1) by AMP-activated protein kinase connects energy sensing to mitophagy. Science 331, 456-461

24. Mihaylova MM and Shaw RJ (2011) The AMPK signalling pathway coordinates cell growth, autophagy and metabolism. Nat Cell Biol 13, 1016-1023

25. Mirouse $V$ and Billaud M (2011) The LKB1/AMPK polarity pathway. FEBS Lett 585, 981-985

26. Al-Rashed F, Calay D, Lang M et al (2018) Celecoxib exerts protective effects in the vascular endothelium via COX-2-independent activation of AMPK-CREB-Nrf2 signalling. Sci Rep 8, 6271

27. Otero-Rodiño C, Velasco C, Álvarez-Otero R et al (2017) Changes in the levels and phosphorylation status of Akt, AMPK, CREB and FoxO1 in hypothalamus of rainbow trout under conditions of enhanced glucosensing activity. J Exp Biol 220, 4410-4417

28. Cho IJ, Woo NR, Shin IC et al (2009) H89, an inhibitor of PKA and MSK, inhibits cyclic-AMP response element binding protein-mediated MAPK phosphatase-1 induction by lipopolysaccharide. Inflamm Res 58, 863-872

29. Wang D, Zhang Y, Lu J et al (2016) Cordycepin, a natural antineoplastic agent, induces apoptosis of breast cancer cells via caspase-dependent pathways. Nat Prod Commun 11, 63-68

30. Hwang JH, Park SJ, Ko WG et al (2017) Cordycepin induces human lung cancer cell apoptosis by inhibiting nitric oxide mediated ERK/Slug signaling pathway. Am J Cancer Res 7, 417-432

31. Su NW, Wu SH, Chi CW et al (2017) Metronomic cordycepin therapy prolongs survival of oral cancer- bearing mice and inhibits epithelial-mesenchymal transition. Molecules 22, E629

32. Cao HL, Liu ZJ, and Chang Z (2017) Cordycepin induces apoptosis in human bladder cancer cells via activation of $A 3$ adenosine receptors. Tumour Biol 39, 1010428317706915

33. Lee EJ, Kim WJ, and Moon SK (2010) Cordycepin suppresses TNF-alpha-induced invasion, migration and matrix metalloproteinase-9 expression in human bladder cancer cells. Phytother Res 24, 1755-1761

34. Yokoyama U, Iwatsubo K, Umemura M et al (2013) The prostanoid EP4 receptor and its signaling pathway. Pharmacol Rev 65, 1010-1052

35. Bastien L, Sawyer N, Grygorczyk R et al (1994) Cloning, functional expression, and characterization of the human prostaglandin $E_{2}$ receptor EP2 subtype. J Biol Chem 269, 11873-11877

36. Funahashi K, Cao X, Yamauchi M et al (2009) Prostaglandin $\mathrm{E}_{2}$ negatively regulates $\mathrm{AMP}$-activated protein kinase via protein kinase A signaling pathway. Prostaglandins Other Lipid Mediat 88, 31-35 